\title{
Il dosaggio del FGF23 con metodica automatizzata: un'esperienza monocentrica nella malattia renale cronica
}

\author{
Caterina Pelosini ', Teresa Lucchese 2, Claudia Mannucci², \\ Claudia D'Alessandro², Roberta Centoni' e Maria Rita Sessa'
}

\begin{abstract}
FGF23 dosage with an automated assay: a single-center experience in patients with renal chronic disease

A key pathogenic role in the development of secondary hyperparathyroidism in renal patients is played by the fibroblast growth factor 23 (FGF23), a $32 \mathrm{kDa}$ protein secreted by osteocytes in response to dietary phosphorus load. FGF23 elicits a phosphaturic response and inhibits calcitriol synthesis. Measurement of FGF23 levels in patients with chronic renal insufficiency can be useful to better define abnormalities of calcium-phosphate metabolism as well as to select those patients in need of dietary interventions targeted to reduce phosphorus intake. Unfortunately, up to now some limits in its application as well as economic factors have prevented the routine use of FGF23 quantification in clinical practice.

The aim of our study was to evaluate the circulating levels of FGF23 in patients with chronic kidney disease using the LIAISON® FGF23 (DiaSorin) assay, a new automated chemiluminescence method that measures the intact molecule and has been approved for clinical use.

Twenty-three consecutive patients ( 14 males and 9 females, with mean age $72.3 \pm 10.9$ years) with stage 3a-5ND CKD were enrolled. The FGF23 assay was also tested in 10 random patients with end-stage renal disease on hemodialysis.

Serum levels of FGF23, PTH, 25(OH)VitD, calcium, phosphorus, creatinine and albumin were measured under fasting conditions.

Within the CKD group, FGF23 serum levels were higher than normal in 16 cases.

Serum FGF23 levels correlated significantly with serum phosphorus $(r=0.600, p<0.01)$, calcium $(r=-0.546, p<0.05)$ and eGFR $(r=-0.495, p<0.05)$. In particular, the negative relationship between residual renal function and circulating FGF23 was significant in patients with eGFR $<30 \mathrm{~mL} / \mathrm{min} / 1.73 \mathrm{mq}(\mathrm{r}=-0.636, \mathrm{p}<0.05)$ but not in those with eGFR $>30 \mathrm{~mL} /$ $\mathrm{min} / \mathrm{l} .73 \mathrm{mq}$.

Although from a small cohort of patients, our data confirm that FGF23 levels increase already in the early stages of CKD; they also confirm that when eGFR levels are $<30 \mathrm{~mL} / \mathrm{min} / \mathrm{I} .73 \mathrm{mq}$ FGF23 increases rapidly and linearly, reaching extremely high levels in the dialysis population.

The availability of an automated assay quantifying the amounts of intact FGF23 molecule, and approved for clinical use by the European Community, represents a valuable tool for obtaining an optimal pharmacological and dietary management of patients with CKD-MBD since the earliest stages of renal disease.
\end{abstract}

\section{Keywords}

FGF23, CKD, Assay, Phosphate, CKD-MBD

\footnotetext{
ISD Laboratorio Chimica e Endocrinologia, Dipartimento di Medicina di Laboratorio, Azienda Ospedaliero-Universitaria Pisana, Pisa, Italy ${ }^{2}$ Dipartimento di Medicina Clinica e Sperimentale, Università di Pisa, Pisa, Italy
}

\section{Corresponding author:}

Caterina Pelosini, SD Laboratorio Chimica e Endocrinologia,

Dipartimento di Medicina di Laboratorio, Azienda Ospedaliero-

Universitaria Pisana, Pisa, Italy.

Email: caterina.pelosini@ao-pisa.toscana.it 


\section{Introduzione}

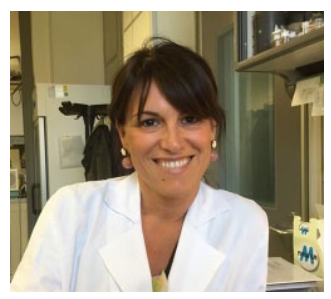

La CKD-MBD (chronic kidney disease - mineral bone disorders) comprende le alterazioni biochimiche del metabolismo minerale e osseo che si manifestano nel corso della malattia renale cronica e le relative complicanze a carico dell'osso e del sistema cardiovascolare. La CKD-MBD include quindi modificazioni dei livelli circolanti di vitamina $\mathrm{D}$, calcio, fosforo e paratormone $(\mathrm{PTH})$, alterazioni del turnover e della mineralizzazione ossea, e calcificazioni vascolari e dei tessuti molli. ${ }^{1}$

La fisiopatologia della CKD-MBD è complessa e caratterizzata inizialmente da meccanismi di compenso omeostatico che coinvolgono rene, intestino, ed osso, ma che poi diventano essi stessi elemento di patologia.

In passato le alterazioni del metabolismo dell'osso nell'insufficienza renale venivano attribuite al deficit di calcitriolo e all'iperparatiroidismo secondario conseguenti alla riduzione della funzione renale. Recentemente un ruolo patogenetico chiave è stato attribuito al fattore di crescita dei fibroblasti 23 (FGF23) e al carico dietetico di fosforo., ${ }^{2,3}$

L'FGF23 è una fosfatonina di $32 \mathrm{kDa}$, secreta dagli osteociti ${ }^{4}$, i cui livelli circolanti aumentano nei pazienti con conseguente riduzione della funzione renale a fronte di un carico dietetico di fosforo. ${ }^{5,6}$ L'FGF23 appartiene alla famiglia dei fattori di crescita dei fibroblasti (FGF), un gruppo di 22 molecole raggruppabili in 7 sottofamiglie che condividono la capacità di legare uno dei quattro recettori specifici (FGFR 1-4) con meccanismo paracrino. ${ }^{7}$ L'FGF23 intatto rappresenta la forma biologicamente attiva, ha un'emivita di circa un'ora ed è metabolizzato nei suoi frammenti inattivi C-terminale e N-terminale. ${ }^{8}$ L'FGF23 esplica la sua azione mediante il legame con il suo recettore e con la proteina transmembrana Tm-Klotho la quale, agendo da co-recettore cellulare, determina l'attivazione della cascata di segnale MAPK/ERK. ${ }^{9}$ Klotho è presente in due isoforme: la forma transmembrana e la forma solubile (s-Klotho). La prima funge da co-recettore per FGF23 ed è espressa solo a livello del tubulo renale distale, delle cellule paratiroidee e del plesso coroideo; la seconda deriva dal clivaggio del dominio extracellulare della forma transmembrana di Klotho. ${ }^{10}$ Klotho ha un'azione fosfaturica indipendente da FGF23 in quanto inibisce il co-trasportatore $\mathrm{Na} / \mathrm{p} 2 \mathrm{a}$ a livello del tubulo renale prossimale ${ }^{11}$ e determina un aumento del riassorbimento di calcio attivando i canali calcio-selettivi apicali a livello del tubulo renale distale.

I principali effetti di un aumento acuto dei livelli circolanti di FGF23 sono l'incremento della fosfaturia e la riduzione della sintesi di calcitriolo. ${ }^{12}$ La prima avviene grazie all'inibizione dell'attività del co-trasportatore sodio/ fosfato Npt2a e Npt2c, localizzato a livello del tubulo prossimale renale; $;^{13}$ la seconda attraverso l'inibizione dell'espressione della 1 - $\alpha$-idrossilasi e l'aumento della 24-idrossilasi. Altra conseguenza di un aumento acuto della produzione di FGF23, è l'inibizione della sintesi e secrezione di PTH da parte delle cellule paratiroidee. ${ }^{10}$

La persistenza di elevati livelli sierici di fosforo e di FGF23 correla con un elevato rischio di mortalità principalmente cardiovascolare. ${ }^{14-16}$ L'associazione tra aumentati livelli di FGF23 ed eventi avversi cardiovascolari è indipendente dai livelli sierici di fosforo, suggerendo che FGF23 e fosforo possano promuovere separatamente meccanismi di tossicità cardiovascolare. ${ }^{17} \mathrm{E}$ ' stato dimostrato che l'aumento della fosforemia è implicato nella disfunzione endoteliale e nelle calcificazioni vascolari, mentre elevati livelli di FGF23 si associano allo sviluppo di ipertrofia ventricolare sinistra, probabilmente con meccanismo diretto indipendente dalla fosforemia. ${ }^{18,19}$ I livelli di FGF23 aumentano già nello stadio 2 di CKD, cioè con un VFG di $60-90 \mathrm{~mL} / \mathrm{min}$, prima ancora che si verifichino alterazioni nei valori ematici di fosforemia e PTH. ${ }^{20,21}$ Tale incremento nelle fasi iniziali di riduzione della funzione renale è considerato un meccanismo compensatorio per prevenire un bilancio positivo del fosforo, l'incremento della fosforemia e del PTH a fronte di un carico dietetico di fosforo. ${ }^{5}$ Con l'ulteriore declino della funzione renale residua, si riducono anche i livelli di Klotho determinando una resistenza periferica all'azione di FGF23 con ulteriore incremento dei livelli circolanti di quest'ultimo. ${ }^{14,21}$ Da questo si comprende come la modulazione dell'apporto dietetico di fosforo rappresenti un momento centrale della prevenzione e cura della CKD-MBD.

Determinare la concentrazione di FGF23 nel paziente con insufficienza renale, può avere una grande utilità per meglio definire lo status del metabolismo fosfo-calcico, identificando anche i pazienti ai quali intensificare una riduzione dell'effettivo apporto di fosforo con la dieta. ${ }^{22,23}$ Limitazioni di ordine metodologico ed economico hanno però impedito fino ad oggi l'uso routinario del dosaggio del FGF23 nella pratica clinica.

I test immunoenzimatici, utilizzati fino ad oggi a fini di ricerca, forniscono valori di FGF23 derivati dalla somma della porzione C-terminale e della forma intatta. Studi più recenti mostrano l'utilità di un nuovo metodo automatizzato in chemilumiscenza per la misurazione della molecola intatta; tale metodo è stato approvato per uso clinico. ${ }^{24}$

Scopo del nostro studio è stato di valutare i livelli circolanti di FGF23 in pazienti affetti da malattia renale cronica in follow-up specialistico, utilizzando il test LIAISON ${ }^{\circledR}$ FGF23 (DiaSorin S.p.a - Saluggia, VC, Italia).

\section{Pazienti e metodi}

Sono stati inclusi nello studio 23 pazienti (14 maschi e 9 femmine, di età media $72.3 \pm 10.9$ anni) clinicamente stabili, con diagnosi di malattia renale cronica allo stadio 
Tabella I. Caratteristiche dei pazienti con CKD stadio 3a-5ND.

\begin{tabular}{|c|c|c|c|c|c|}
\hline & Totale media & DS & mediana & QI & Q3 \\
\hline Età (anni) & 72.3 & 10.9 & 76.0 & 66.5 & 78.0 \\
\hline Peso (kg) & 75.3 & 16.9 & 72.0 & 64.3 & 82.0 \\
\hline IMC (kg/mq) & 26.4 & 5.0 & 26.1 & 23.8 & 28.3 \\
\hline Creatininemia (mg/dL) & 2.2 & 1.3 & 1.7 & 1.6 & 2.3 \\
\hline eGFR-EPI (mL/min/I.73mq) & 32.1 & 12.5 & 29.3 & 23.8 & 40.3 \\
\hline FGF23 (pg/mL) & |58.| & 127.4 & 121.7 & 74.9 & 195.2 \\
\hline Calcemia (mg/dL) & 9.4 & 0.5 & 9.4 & 9.2 & 9.7 \\
\hline Fosforemia (mg/dL) & 3.2 & 0.8 & 3.0 & 2.6 & 3.6 \\
\hline PTH (pg/mL) & 71.8 & 44.5 & 62.0 & 37.0 & 95.0 \\
\hline 25(OH)VitD (ng/mL) & 27.5 & 13.2 & 27.4 & 21.1 & 37.0 \\
\hline Albuminemia (g/dL) & 4.1 & 0.5 & 4.2 & 3.8 & 4.4 \\
\hline
\end{tabular}

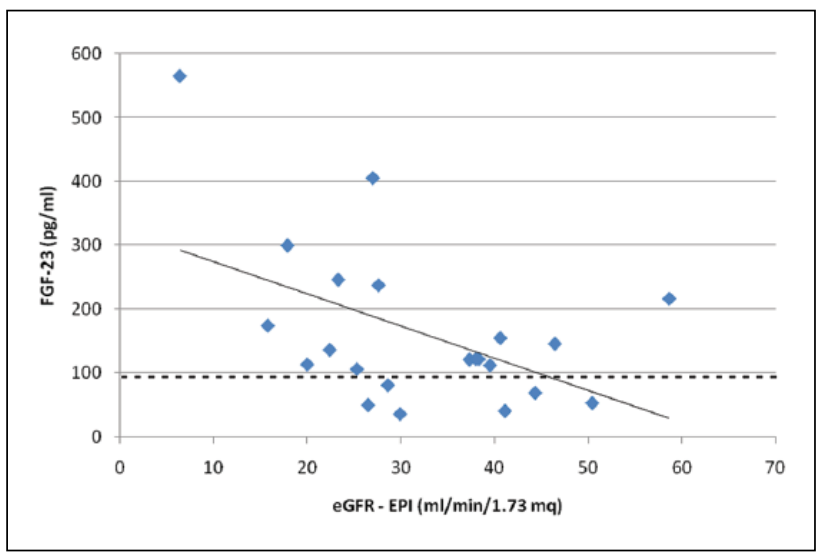

Figura I. Correlazione fra livelli di FGF23 e di eGFR nei pazienti con CKD. La linea tratteggiata rappresenta il limite alto di normalità dell'FGF23.

3a-5ND, quindi con eGFR $<60 \mathrm{~mL} / \mathrm{min} / 1.73 \mathrm{mq}$, e seguiti presso l'ambulatorio nefrologico dedicato alla malattia renale cronica.

Sono stati assunti i seguenti criteri di esclusione: età $<18$ anni, terapie in atto con cinacalcet, calcitriolo o paracalcitolo o chelanti intestinali del fosforo. Sono stati altresì esclusi pazienti reduci da meno di 6 mesi da interventi chirurgici, o quelli con malattie infiammatorie intestinali, malattie infettive o infiammatorie in fase acuta, o affetti da neoplasie in fase avanzata.

Per ciascun paziente sono stati misurati, in condizioni di digiuno, i livelli circolanti di FGF23, paratormone, 25(OH) VitD, calcemia, fosforemia, creatininemia e albuminemia. La stima del filtrato glomerulare è stata effettuata usando la creatininemia secondo l'equazione eGFR-EPI.

Per il dosaggio dell'FGF23 intatto è stato utilizzato il kit LIAISON® (DiaSorin S.p.a - Saluggia, VC, Italia), un test sandwich a tre step basato sul principio della chemiluminescenza.

Il PTH è stato determinato con metodica di terza generazione LIAISON ${ }^{\circledR} \quad 1-84$ PTH. L'intervallo di riferimento per l'FGF23 è compreso tra $23.2 \mathrm{pg} / \mathrm{mL}$ e 95.4 $\mathrm{pg} / \mathrm{mL}$ e per il iPTH tra $8 \mathrm{ng} / \mathrm{L}$ e $40 \mathrm{ng} / \mathrm{L}$, secondo le indicazioni riportate nelle istruzioni per l'uso.

Il metodo è stato testato anche in 10 pazienti random in trattamento emodialitico cronico.

\section{Analisi statistica}

I risultati sono espressi come media \pm deviazione standard (DS) e l'analisi statistica è stata effettuata mediante test t di Student e correlazione lineare di Pearson. Valori di $\mathrm{p}<0.05$ sono stati considerati come statisticamente significativi.

\section{Risultati}

Sedici dei 23 pazienti inclusi nello studio presentavano livelli di FGF23 superiori alla norma in associazione con valori di creatininemia compresi tra $1.3 \mathrm{mg} / \mathrm{dL}$ e $7.3 \mathrm{mg} /$ $\mathrm{dL}$ e con un eGFR compreso tra 6.4 e $58 \mathrm{~mL} / \mathrm{min} / 1.73 \mathrm{mq}$.

Ventidue pazienti su 23 mostravano valori di fosforemia che rientravano nel range di normalità, e solo un paziente (in stadio 5ND) era iperfosforemico. Dei 23 pazienti, 7 avevano valori di iPTH nel range di normalità, mentre $i$ restanti mostravano valori di paratormone elevati (fino a $158 \mathrm{pg} / \mathrm{mL}) ; 2$ pazienti su 23 avevano una calcemia inferiore a $8.6 \mathrm{mg} / \mathrm{dL}$ (tabella 1 ).

Nei pazienti con CKD, i livelli sierici di FGF23 correlavano con fosforemia $(r=0.600, p<0.01)$, calcemia $(\mathrm{r}=-0.546, \mathrm{p}<0.05)$ ed eGFR $(\mathrm{r}=-0.495, \mathrm{p}<0.05)$. In particolare, nei pazienti con eGFR $<30 \mathrm{~mL} / \mathrm{min} / 1.73 \mathrm{mq}$, la correlazione negativa tra funzione renale residua ed FGF23 circolante risultava significativa $(r=-0.636$, $\mathrm{p}<0.05$ ) (figura 1); tale correlazione risultava meno evidente per valori di eGFR $>30 \mathrm{~mL} / \mathrm{min} / 1.73 \mathrm{mq}$.

La correlazione tra valori di FGF23 ed altri parametri quali età, livelli di PTH, ed albuminemia non ha mostrato alcuna significatività statistica nei pazienti di questo studio.

Nel campione di 10 pazienti in trattamento emodialitico trisettimanale ( 8 maschi e 2 femmine) con età compresa tra 
Tabella 2. Caratteristiche dei pazienti in emodialisi.

\begin{tabular}{lrrrrr}
\hline & totale media & DS & mediana & QI & Q3 \\
\hline Età (anni) & 60.5 & 17.4 & 62.5 & 48.5 & 73.8 \\
Peso (kg) & 72.3 & 15.5 & 75.5 & 67.3 & 77.9 \\
FGF23 (pg/mL) & $119,807.0$ & 196090.0 & $27,718.0$ & $17,525.0$ & $65,138.5$ \\
Calcemia (mg/dL) & 8.7 & 0.5 & 8.8 & 8.5 & 4.0 \\
Fosforemia (mg/dL) & 5.3 & 1.3 & 4.9 & 5.7 \\
PTH $(\mathbf{p g} / \mathbf{m L})$ & 282.1 & 210.6 & 166.5 & 164.0 & 355.5 \\
\hline
\end{tabular}

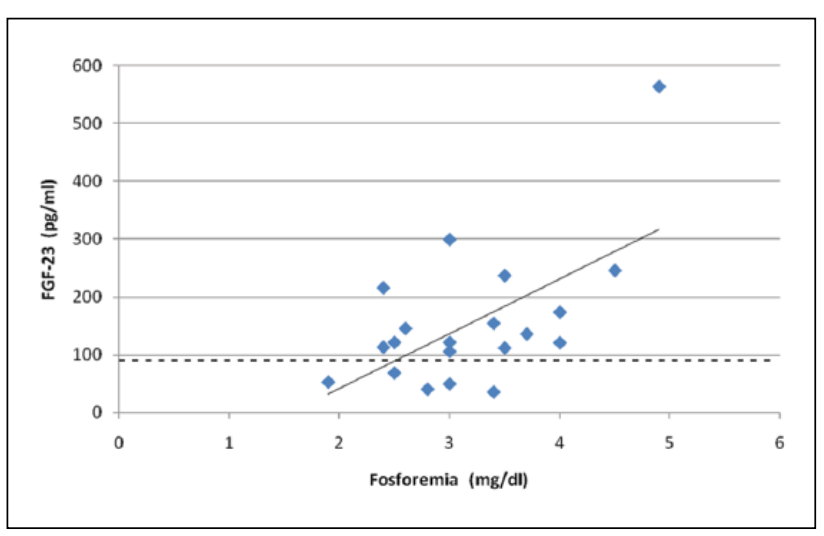

Figura 2. Correlazione tra fosforemia e FGF23 nei pazienti con CKD. La linea tratteggiata rappresenta il limite alto di normalità del FGF23.

i 31 e gli 85 anni, i valori di FGF23 risultavano aumentati di circa 3 ordini di grandezza (tabella 2).

\section{Discussione}

La nostra esperienza, seppur relativa ad un campione di ridotte dimensioni, è in linea con quanto riportato in letteratura riguardo al dosaggio di FGF23 nei pazienti nefropatici. ${ }^{24}$

Nei pazienti con CKD in terapia conservativa, i nostri dati hanno evidenziato una correlazione negativa tra $\mathrm{i}$ valori di FGF23 e l'eGFR-EPI. Fin dalle prime fasi di riduzione della funzione renale, i livelli sierici di FGF23 aumentano, ma è a partire dallo stadio 4 di malattia che si assiste ad un più rapido e progressivo incremento (figura 1). Nei pazienti in emodialisi i valori sierici di FGF23 risultavano straordinariamente elevati, di circa 3 ordini di grandezza superiori rispetto a quelli riscontrati nei pazienti con CKD non in dialisi. Alcuni dati della letteratura suggeriscono che 1'incremento del FGF23 sia legato ai seguenti fattori: un'aumentata produzione o una ridotta clearance metabolica, la resistenza tissutale dovuta alla diminuzione dei livelli di Tm-Klotho, l'iperfosforemia, e l'iperparatiroidismo secondario; non si può del resto escludere che anche altri fattori o tossine uremiche possano contribuire all'aumento dei livelli di FGF23.
L'incremento di FGF23 intatto rappresenta inizialmente un meccanismo compensatorio per prevenire la comparsa di iperfosforemia e iperparatiroidismo secondario nelle prime fasi di CKD-MBD. Questo si ritrova anche nell'analisi dei nostri dati, dove i pazienti con normale fosforemia presentano valori di FGF23 già aumentati rispetto al range di normalità, in accordo con quanto presente in letteratura (figura 2). ${ }^{25}$

E' stato riportato che nei pazienti con riduzione della funzione renale, la restrizione dietetica di fosforo associata all'impiego dei chelanti del fosforo è in grado di determinare una riduzione dei livelli di FGF23 se assunti per due settimane, mentre un trattamento prolungato non sembra produrre lo stesso effetto., ${ }^{6-28}$ Una notevole importanza è stata attribuita anche alla fonte di fosforo: pazienti che seguono una dieta vegetariana mostrano livelli significativamente ridotti di FGF23 e di fosforemia rispetto a quelli riscontrati in pazienti che seguono una dieta a base di carne, nonostante l'intake di proteine e di fosforo fosse uguale nei due gruppi. ${ }^{29}$ Tuttavia, alcuni studi che hanno esaminato l'effetto dell'intake di fosforo con la dieta sui livelli circolanti di FGF23 non hanno evidenziato un legame diretto tra i due parametri, suggerendo la possibilità che esista una correlazione indiretta mediata dalla vitamina D o dalla mineralizzazione ossea. ${ }^{30,31}$

Come descritto in letteratura, in fase acuta l'FGF23 inibisce la sintesi e la secrezione di PTH; in fase cronica questo effetto è superato dal deficit di calcitriolo, noto stimolo per la sintesi e secrezione del PTH. L'FGF23 sopprime inoltre la trascrizione del gene di Klotho a livello renale e la produzione di calcitriolo: ne consegue una riduzione della calcemia, che può spiegare la correlazione negativa tra FGF23 e calcio. ${ }^{10,32,33}$

Anche i livelli di calcemia sembrano a loro volta regolare quelli di FGF23: in condizioni di ipocalcemia, infatti, il calcio può bloccare la secrezione dell'FGF23 indotta da fosforo e PTH, come meccanismo di compenso ad un ulteriore riduzione del calcitriolo, che potrebbe quindi esacerbare l'ipocalcemia.

Il test LIAISON ${ }^{\circledR}$ FGF23 utilizzato in questo studio è una metodica in chemiluminescenza completamente automatizzata che utilizza tre anticorpi monoclonali. Il primo anticorpo è legato a microparticelle e diretto contro la porzione $\mathrm{N}$-terminale della molecola intatta; il secondo 
è marcato con fluoresceina, e diretto contro la porzione C-terminale; il terzo è marcato con isoluminolo e diretto contro la fluoresceina. Nelle istruzioni per l'uso del produttore è dichiarata una specificità del test del $100 \%$ verso la forma intatta del FGF23 con assenza di interferenza verso altre forme di FGF ed un LOQ (limit of quantification) $<10 \mathrm{pg} / \mathrm{mL}$ confermato dalla letteratura. ${ }^{24}$ Il test LIAISON ${ }^{\circledR}$ FGF23 mostra un'ottima correlazione con i valori ottenuti utilizzando il saggio Human FGF23 (Intact) ELISA Immutopics (Immutopics Inc. San Clemente CA92673), che è stato a lungo il più utilizzato nella pratica di laboratorio (dati personali, non pubblicati); tuttavia si tratta di un saggio utilizzato esclusivamente nel campo della ricerca e, a differenza del test utilizzato in questo studio, non è completamente automatizzato. Per queste ragioni il test da noi impiegato presenta indubbi vantaggi rispetto alle metodiche immunoenzimatiche sia dal punto di vista della gestione del dosaggio che dal punto di vista della sensibilità e della riproducibilità dei risultati.

\section{Conclusioni}

I nostri dati, benchè riferiti ad una piccola coorte di pazienti, confermano che i livelli di FGF23 aumentano nei primi stadi della malattia renale cronica, e confermano che al di sotto di un eGFR di $30 \mathrm{~mL} / \mathrm{min} / 1.73 \mathrm{mq} \mathrm{l'FGF23}$ aumenta in modo rapido e lineare fino a raggiungere livelli estremamente elevati nella popolazione in dialisi.

La disponibilità di una metodica automatizzata per il dosaggio di FGF23 intatto, approvata per l'utilizzo clinico da parte della Comunità Europea, può rappresentare un valore aggiunto al fine di ottenere una gestione ottimale del paziente affetto da CKD-MBD a partire dalla fase predialitica, dove il controllo e la riduzione dell'apporto dietetico di fosforo rappresentano gli elementi iniziali e centrali della prevenzione e trattamento della CKD-MBD.

\section{Dichiarazione di assenza di conflitto di interessi}

Gli Autori dichiarano di non avere conflitti di interessi.

\section{Finanziamenti}

Gli Autori dichiarano di non aver ricevuto finanziamenti specifici da qualsiasi ente nei settori pubblico, privato o senza fini di lucro.

\section{Bibliografia}

1. Moe SM, Drueke T, Lameire N, et al. Chronic kidney disease-mineral-bone disorder: a new paradigm. $A d v$ Chronic Kidney Dis 2007; 14:3-12.

2. Martin A, David V and Quarles LD. Regulation and function of the FGF23/Klotho endocrine pathways. Physiol Rev 2012; 92:131-155.

3. Komaba H and Fukagawa M. FGF23: a key player in mineral and bone disorder in CKD. Nefrologia 2009; 29(5):392-396.

4. Stubbs J, Liu S and Quarles LD. Role of fibroblast growth factor 23 in phosphate homeostasis and pathogenesis of disordered mineral metabolism in chronic kidney disease. Seminars in dialisi 2007; 20:302-308.

5. Antoniucci DM, Yamashita $\mathrm{T}$ and Portale AA. Dietary phosphorus regulates serum fibroblast growth factor-23 concentration in healthy men. $J$ Clin Endocrinol Metab 2006; 91:3144-3149.

6. Isakova T, Barchi-Chung A, Enfield G, et al. Effects of dietary phosphate and phosphate binders on FGF23 levels in CKD. Clinical journal of the American Society of Nephrology: CJASN 2013; 8(6):1009-1018.

7. Mohammadi M, Olsen SK and Ibrahimi OA. Structural basis for fibroblast growth factor receptor activation. Cytokine Growth Factor Rev 2005; 16:107-137.

8. Imanishi Y, Inaba M, Nakatsuka K, et al. FGF-23 in patients with end stage renal disease on hemodialysis. Kidney Int 2004; 65:1943-1946.

9. Urakawa I, Yamazaki Y, Shimada T, et al. Klotho converts canonical FGF receptor into a specific receptor for FGF23. Nature. 2006; 444:770-774.

10. Ben-Dov IZ, Galitzer H, Lavi- Moshayoff V, et al. The parathyroid is a target organ for FGF23 in rats. J Clin Invest 2007; 117:4003-4008.

11. Erben RG. Update on FGF23 and Klotho signaling. Mol and Cell Endocrinology 2016;432:56-65.

12. Kuczera P, Adamczak M and Wiecek A. Fibroblast Growth Factor-23. A potential Uremic Toxin. Toxins. 2016; 8:369.

13. Yamashita T. Structural and biochemical properties of fibroblast growth factor 23. Ther Apher Dial 2005; 9: 313-318.

14. Kovesdy CP and Quarles LD. The role of fibroblast growth factor-23 in cardiorenal syndrome. Nephron Clin Pract 2013; 123(3-4):194-201.

15. Munoz Mendoza J, Isakova T, Ricardo AC, et al. Fibroblast growth factor 23 and Inflammation in CKD. Clinical journal of the American Society of Nephrology: CJASN 2012; 7(7):1155-1162.

16. Gutiérrez OM, Januzzi JL, Isakova $\mathrm{T}$, et al. Fibroblast growth factor 23 and left ventricular hypertrophy in chronic kidney disease. Circulation 2009; 119(19):2545-2552.

17. Pasquali M, Tartaglione L and Rotondi S. I nuovi biomarcatori della CKD-MBD. Giornale italiano di nefrologia 2016; 33(6):1724-5590.

18. Faul C, Amaral AP, Oskouei B, et al. FGF23 induces left ventricular hypertrophy. J Clin Invest 2011; 121(11): 43934408.

19. Scialla JJ, Asplin J, Dobre M, et al. Higher net acid excretion is associated with a lower risk of kidney disease progression in patients with diabetes. Kidney Int 2017; 91(1):204-215.

20. Isakova $\mathrm{T}$, Wahl $\mathrm{P}$, Vargas GS, et al. Fibroblast growth factor 23 is elevated before parathyroid hormone and phosphate in chronic kidney disease. Kidney international 2011; 79(12):1370-1378.

21. Wolf M. Update on fibroblast growth factor 23 in chronic kidney disease. Kidney International 2012; 82(7):737-747.

22. Cupisti A and Kalantar-Zadeh K. Management of natural and added dietary phosphorus burden in kidney disease. Semin Nephrol 2013; 33(2):180-190.

23. D'Alessandro C, Piccoli GB and Cupisti A. The "phosphorus pyramid": a visual tool for dietary phosphate management in dialysis and CKD patients. BMC Nephrol 2015; 16:9. 
24. Souberbielle JC, Prié D, Cavalier E, et al. Evaluation of a New Fully Automated Assay for plasma Intact FGF23. Calcif Tissue Int 2017; 101(5):510-518.

25. Ezumba I, Quarles LD and Kovesdy CP. FGF23 e cuore. Gionrale Ital Nefrol 2014; 31(6).

26. Goto S, Nakai K, Kono K, et al. Dietary phosphorus restriction by a standard low-protein diet decreased serum fibroblast growth factor 23 levels in patients with early and advanced stage chronic kidney disease. Clinical and experimental nephrology 2014; 18(6):925-931.

27. Wan-Chuan Tsai, Hon-Yen Wu, YU-Sen Peng, et al. Effects of lower versus higher phosphate diets on fibroblast growth factor-23 levels in patients with chronic kidney disease: a systematic review and meta-analysis. Nephrol Dial Transplant 2018; 33(11):1977-1983.

28. Saito H, Maeda A, Ohtomo S, et al. Circulating FGF23 is regulated by 1alpha, 25-dihydroxyvitamin D3 and phosphorus in vivo. J Biol Chem 2005; 280(4):2543-2549.
29. Moe SM, Zidehsarai Mp, Chambers MA, et al. Vegetarian compared with meat dietary protein source and phosphorus homeostasis in chronic kidney disease. Clin J Am Soc Nephrol 2011; 6:257-264.

30. Vervloet MG, van Itterrsum FJ, Buttler RM, et al. Effects of dietary phosphate and calcium intake on fibroblast growth factor-23. Clin J Am Soc Nephrol 2011; 6:383-389.

31. Sigrist M. Tang, et al. Responsiveness of FGF23 and mineral metabolism to altered dietary phosphate intake in chronic kidney disease (CKD): results of a randomized trial. Nephrol Dial Transpl 2013; 28:161-169.

32. Galitzer H, Ben-Dov IZ, Silver J, et al. Parathyroid cell resistance to fibroblast growth factor 23 in secondary hyperparathyroidism of chronic kidney disease. Kidney Int 2010; 77:211-218.

33. Rodriguez-Ortiz ME, Lopez I, Muñoz-Castañeda JR, et al. Calcium deficiency reduces circulating levels of FGF23. $J$ Am Soc Nephrol 2012; 23(7):1190-1197. 